\section{THE CROONIAN LECTURES}

\section{INFLUENCE OF THE CIRCULATION UPON THE NERVOUS SYSTEM.}

\section{Delivered before the Royal College of Physicians.}

By WALTER MOXON, M.D., F.R.C.P., Physician to Guy's Hospita!.

\section{LECTURE II.-PART II.}

IF, indeed, we cannot prove by post mortem examination that a person has died from congestion of the brain, the evidence which would lead us to declare the existence of such a state during the person's life ought to be the more conclusive. Let us now inquire if this is truly the case. I have already asked your attention to the degree of turgescence of the veins of the head and face indicating a like degree of pressure within the veins of the cranium, which is met with frequently under various circum. stances, and which does not give rise to serious or important symptoms; strangulation becomes very decided and obvious before any serious cerebral effects result from it. Hence we must not suppose or assume its pressure unless we can see the veins evidently turgid; and although total stoppage of the jugular veins by ligature will produce speedy death, yet, inasmuch as we thereby deprive the brain of its blood-circulation, so that life ceases with the cessation of vital changes in the respiratory centres, se have here a set of consequences which ensure death, apart from pressure of venous blood upon the brain. Indeed, I have never met with any example in which venous pressure upon the brain, as distinguished from the effects of carbonic acid poisoning, has produced grave symptoms. And I advanced the example of caisson-pressure as affording an instance of extreme degrees of intravascular pressure of blood upon the nervous centres uncomplicated with a venous state of the blood; and it was shown that, under a pressure of three atmospheres, continued for as many hours, no unpleasant results were experienced; so far otherwise, indeed, that the labourers worked more buoyantly in the denser air. It was when the pressure was taken off that trouble came. As one more example to show how well an obvious amount of arterial pressure is borne by the brain, let us take the case of unusually violent action of an hypertrophied heart. It is not, of course, to be supposed that in all or most cases of cardiac hypertrophy an excessive action of the heart would tend to congest the brain, because for the most part the heart enlarges in order to overcome an obstruction at its orifices or an obstruction in the capillary system. In these cases, the hypertrophy does but follow after, and usually far after, the requirement which it subserves, and the large labouring heart is all too feeble for its extra work. But we meet occasionally with more rare examples, wherein a large heart certainly does appear to exert an exaggerated force throughout the vessels. A striking example of this is in the Clinical Ward at Guy's Hospital, in the case of a man of early middle age, who has regurgitant aortic valves and a very greatly hypertrophied heart, which raises the præcordial region, and in its beating shakes forcibly the lower part of the chest. The innervation of the heart is here deranged, as if by a touch of exophthalmic cachexia superadded; for the heart's action is very rapid, and digitalis, in any doses I dare give, shows no power of reducing its frequency. The effect of all this in the temporal and radial arteries is remarkable; they are bent into a meandering form, and are tense to touch, each beat of the heart making them spring rather sharply. Those who witriessed the force evidently at work within these arteries, and conceived the same action as going on within the cranium, formed the impression that the arterial strain must be excessive there, allowing all that the bend of the carotid canal could do to moderate the distension of the arteries in their transmission through the base of the skull. Yet this man said that his head was quite free from unpleasant sensations; and, when I induced him to cough, and otherwise to use strong pressure of his expiratory muscles, so that the veins rose in his neck, the heart beating, meantime, still more strongly and quickly with his exertion, no ill effects occurred in the way of morbid sensations in the brain. This example is not a very rare one, although the coexistence of rapid action of the heart, with great hypertrophy and absence of evidence of aortic obstruction, offered a combination calculated to put great stress upon the interior of the vessels, and the strain was visibly great in the superficial arteries. In. deed, under similar circumstances, especially in cases of exophthalmos, we witness an extent of more or less violent and certainly obvious overaction in the cervical vessels, producing no noticeable discomfort in the brain, and certainly nothing of the nature of serious and important symptoms.

Hence, as we pass to consider what clinical evidence there is to show that serious and important diseases are due to cerebral congestion, we do so having first recognised that, visibly, forcible arterial action does not produce these symptoms; and, visibly, great venous congestion does not produce them, and post mortem evidence is incapable of proving that cerebral hyperænia was present before the act of dying. Still pursuing the question before us, the next thing will be to learn what evidence is offered in the symptoms ascribed to cerebral congestion to show that fulness of the cerebral blood-vessels causes the attacks described under that name. If we look at the descriptions of the sup. posed disease, we find it has all the advantage of being well pour. trayed by the best describers, including a most trustworthy and universally trusted authority upon the subject of brain-diseases in general ; so that we may fairly assume that all that can be said for the disorder is well and fully said. The disease would merit such treatment, if it is indeed capable of appearing in no fewer than four distinct and very widely diverse manifestations, and if in each of these it may constitute an obscure and rapidly fatal malady. The symptoms of such a disease must merit the closest attention; and so I may ask you to let me now give a full account of the symptoms described as those of one of the forms of congestion of the brain : it is called the convulsive form, and these are the symptoms given. "There are usually the premonitory signs of congestion, but these may be very slight; there may be no forewarnings, and the patient may be seized during sleep, or while making some unaccustomed effort. When the attack occurs during sleep, it is difficult, and sometimes impossible, to say in what manner it commenced; but when it has come on while the patient is awake, and friends are around him, it has been usually observed that much discomfort has preceded it for a few seconds, minutes, or hours. A tight. cravat worn while making undue exertion, a sudden alarm, or an in. digestible meal rapidly swallowed, may be the immediate antecedents."

"The patient more or less suddenly becomes confused, then apparently half unconscious, makes some unintelligible sounds, turns red and then blue in the face, staggers for support, looks round him wildly and imploringly, and then sits down or falls down convulsed, and a paroxysm, epileptiform in character, supervenes. From this he recovers partially, exhibiting great confusion of mird, headache, muscular feeble. ness, and sometimes partial paralysis of one side or of one limb. The attack is occasionally followed by quasi-maniacal excitement, lasting from half an hour to three or four hours; after which, the patient becomes exhausted and falls into a heavy sleep. From this state he may recover, or, during sleep, a second or third attack of convulsions may come on. When congestion of the brain has assumed this convulsive form, the patients, so far as my own experience extends, have usually been in middle life, and have recovered. But in other cases, when the age has been further advanced, the attacks have recurred more frequently, the intervals between them have become of shorter duration, and the patients-less sensible and less reasonable after every paroxysm-have presented the appearance of those whose attacks have been apoplectiform at their commencement." This description, coming from the pen of a masterly describer, puts vividly before us the condition depicted; but that condition appears so exactly like epilepsy in a plethoric person, that one is rather puzzled to detect a difference between the convulsive form of congestion of the brain on the one hand, and epilepsy in such a person on the other, and one gladly turns for guidance to the directions for diagnosis, which tell us that : "From epilepsy, the diagnosis may be made by a consideration of the previous history; in the one, there have been the premonitory symptoms of con. gestion of the brain; in the other, no such phenomena have presented themselves; in the former, the patient is usually of middle or advanced age; in the latter he is young, and is either uncler twenty years of age, or has not far exceeded that period of life; in the one, the period of most marked congestion is at the moment of seizure; in the other, congestion of the face and head is most marked as the attack is passing off; in the former, there may be some moaning sound; in the latter, the epileptic cry; in the one, there is the suciticn onset of an acute disease ; in the other, the attack of a chronic malady." Thus it appears that the points of distinction in the attack are the middle or advanced age of the patient; the comparison of the colour of his face in the beginning and in the subsequent course of his fit; and the moaning sound in congestion, as compared with the "cpileptic cry" in epilepsy.

As to the latter point, we, however, find the same distinguished authority speaking thus in an admirable description of epilepsy itself : 
"Others do not cry, but emit a moaning sound, which is, as it were, squeezed out of them by the quasi-tonic contraction of the muscles of the chest." Thus the moaning sound does not help us, if we bear in mind the account of true epilepsy. Again, as to age, the same full and excellent account of true epilepsy informs us that a considerable proportion of first attacks occur about the fortieth year of life; so that the age of the patient would appear to be no valid criterion, and then the only thing remaining is the play of colours in the patient's face during the fit. Th.us the convulsive form of congestion resolves itself into an attack which is exactly like epilepsy, except in the plethoric appearance of the patient. But are we then to suppose that plethoric persons do not have epilepsy? If this is not the supposition, how are we to deal with the case of a red-faced person who should have a true epileptic fit? How should we distinguish between such an attack and an attack of congestion of the brain? One looks in vain through the description for any criterion. There are all the stages of epilepsy : the blue tinge appearing on the red of the face; the groan; the fall; the struggle; the stupor; and even the final, and perhaps almost maniacal, excitement at the close. Even the tight cravat and the full meal, which appear in the description of congestion, do not really help us, because the true epileptic might have on a tight cravat, or might have had a full meal. The evidence, then, of congestion of the brain is utterly worthless, unless you assume that those plethoric persons do not have epilepsy; and that, after a full meal or in a tight cravat, epilepsy is not epilepsy. But the question whether such persons have epilepsy is not even raised by the description, which changes the whole fundamental pathology of the seizures because the sufferer's face is red and he has just had his dinner. I have quoted from the writings of one who is so entirely master of the subject of nervous diseases, that the evidences in favour of congestion of the brain would certainly all be fully and fairly stated in his description of it. Yet surely we have seen but very trivial ground for raising the convulsive form of congestion of the brain as a distinct kind of disease; and we further have even some proof of a positive kind to show that you cannot judge of the amount of blood in the brain by the amount of blood in the face. At least, in sleep, we now know the brain to be pale; yet in sleep the face is more congested than it is under ordinary conditions when awake. If you look at a sleeping person, you may commonly observe, everywhere about the head, signs that would show congestion: the contracted pupils; the suffused conjunctiva, often causing an excess of secretion from the mucous surface, or the well-known puffy state of face and eyelids found in the morning in cases of Bright's disease and other conditions; the reddened and perspiring state of the skin conspicuous in many people in deep sleep, and often wetting the pillow in phthisis; yet, in spite of all these signs of congestion outside the cranium, the brain within it is shown to be pale in sleep. So far, then, from redness of face proving congestion of the brain, the best known instance bearing on the question shows the brain to be paler when the face is more congesterl than the average.

The importance of the question whether, as asserted in some textbooks, disease and death are really due to a rush of blood into the vessels of the brain, or whether, as I believe, no serious symptoms or ill results are produced by blood rushing into or pressing within the blood-vessels of the brain, will, I trust, justify me in pursuing the examination of the asserted symptoms of cerebral congestion, still searching for evidence that these symptoms are due to congestion of the brain. Yet, as to the form of the disease, that is called apoplectiform cerebral congestion, I will not go into detail, because the kind of attack of sudden insensibility, which has long been known by this name, has been so thoroughly treated of by the late Professor Trousseau, and by him was so entirely well proved to be really epileptic, that I need only refer for evidence to Trousseau's lecture upon the subject. In that lecture, he says: "During the first years of my practice, I saw a pretty large number of cases of apoplectiform congestion; but for a long time I have not seen any. Yet other medical men see as many as before. Let us, therefore, inquire on whose side the error lies." And then he gives proofs of the epileptic nature of such attack, which I think so convincing that they need only to be read to clear up any doubt upon the matter. It is, perhaps, due to Professor Trousseau's influence that the most modern English teaching is coming round to the same view, as shown in the most recent and one of the best of our textbooks, in which we find it written: "The true apoplectic attack, in which the patient falls down suddenly comatose, is now generally admitted to be epileptic." As a small further contribution to the previous evidence upon the epileptic nature of apoplectiform cerebral congestion, I will only point to one feature in descriptions of the attack; and that is "the pulse at the moment of the attack is sometimes suspended at the wrist". Of course, it is plain that suspension of the pulse would not be likely to produce congestion of the brain: and, indeed, it is clear that suspension of the pulse must cause anæmia of the brain. But I will bring forward some facts in my: next, lecture which will, I trust, show that suspension of the pulse accompanies and marks the onset of attacks of epilepsy, and is the cause of paleness of the face in the epileptic onset. I need hardly say that the question at issue is one that practically is important; for, if the attack is epileptic, it calls upon us for remedies, and requires such as reduce the general excitability of the nervous system, and especially for such specifics as potassium bromide. But if the attack is indeed congestion of the brain, then it calls upon us for local treatment of the head, more or less of the nature of depletion.

If we seek in the two remaining forms for evidence that the disease is really due to congestion of the brain, we are met with proof to show that the contrary is really the case. The characters of the third, or "febrile form", are those of an attack accompanied by marked elevation of temperature and general pyrexia, dull oppressive headache, etc. Recovery is said not always to be observed. "The distress may persist; there may be alternately convulsions and delirium; or there may be changes from overexcitement to drowsiness-the latter gradually becoming relatively more marked until the patient passes into a state of stupor, from which he may never rally." Now, if such a group of symptoms were to come upon a healthy person, it is a fair question whether the medical attendant would be well advised in resting satisfied with an opinion that the case is one of congestion of the brain. If the attack ended fatally, would he not have to inquire which of the eruptive fevers, or what form of blood-poisoning, had seized and killed the patient without allowing time for the development of the distinguishing characters of the attack? In every case in which I have known a healthy person fall into febrile coma, that person has proved to be suffering from a definite disease which needed to be discovered. For example, a little girl at Tottenham, having precisely these symptoms, was said to have died within twenty-four hours of "congestion of the brain". Scarlet fever appeared in her sisters within twenty-four hours more, showing what was the matter. A man approached very near to death, being quite comatose, with elevated temperature; he then developed a most profuse urticaria, and forthwith recovered. Had he died, we should not have known better, perhaps, than to have called his disorder cerebral congestion. Such instances might be multiplied indefinitely. I am ready to admit that such a term as congestion of the brain is useful to the practitioner in some of his difficulties; and, so far as it is understood to signify only that the case to which it is applied is cerebral and obscure, there is no harm in it. Nay, there is a certain advantage in its indefiniteness; whilst it meantime does not usually carry an unduly sudden or alarming impression to the minds of the patient's friends. But, for all this, it is surely undesirable to make this conveniently vague term take a place in strict medical teaching, and to use it there as covering several widely diverse disordered states, which can be only very vaguely pourtrayed even by our best clinical describers, seeing that there must arise a great temptation to stop at this idea of congestion of the brain, when further inquiry would have shown the true nature of the case. It is true that we are compelled to use some terms to give expression to all that we can know of obscure disorders, and it might be thought that "congestion of the brain" stands thus on a par with "sciatica" and "colic"; but there is a wide difference between these at first apparently parallel examples. When a person has pain in the course of his sciatic nerve, we search for aneurysm or other tumour which might cause the pain; and, if we do not find any such cause, we say that the case is one of sciatica. Even here there is a danger of our too easily taking up with the easier diagnosis of sciatica-a danger which not unfrequently induces the practitioner to overlook an organic cause of pain. However, there is nothing wrong in describing pain in the course of the sciatic nerve as sciatica, since the term means only sciatic nerve pain. Likewise, in the case of such an expression as colic, the term applies without doubt, whatever be the nature of the colic pain. The term involves no supposition of a definitive cause of the complaint, and there is nothing positively misleading in the use of it. But in the example of "congestion of the brain", employed in a similar vague way, there is this important difference, that the term does signify a fulness of, and pressure of blood within, the cerebral vessels; which fulness and pressure may not exist, and, I think I have shown, probably do not exist. Thus whereas, in any prepossession with the idea of sciatica, we only incur the risk of sometimes failing to reach the whole truth of individual cases, we are probably encirely wrong at all levels when we use the term congestion of the brain. Again, this term does not only apply to one definite kind of pain like sciatica and colic; but it is made to include convulsive disease, apoplectiform disease, febrile disease, and mental aberration-thus extending its shadow and obscurity to confuse a very large part of the otherwise clear history of diseases of the brain. 
We could not, perhaps, have a better example to show how far the description of these diseases as congestion of the brain is correct, than we meet with under the form I am now discussing - the febrile form of cerebral cangestion-for we see the whole group of symptoms described as the febrile form of cerebral congestion appear as "cerebral rheumatism" in rheumatic fever. Now in cerebral rheumatism, with its perfectly characterised febrile congestion of the brain, we happen to have very decisive evidence that there is no congestion of the brain, but the exact opposite of this. Ten years ago, one did not know what to do with the rapidly fatal brain-symptoms which occur in some cases of rheumatic fever. I tried bleeding, and large doses of brandy and large doses of quinine, but all the patients died. The plan now successfully adopted is well known. I first practised it in 1870, and an account of the case was published by Mr. De Liefde, the housephysician. I mention it because of the somewhat rude thoroughness of the treatment. The patient had passed through the stage of muttering delirium, and was settling into insensibility; when at a temperature of about $106^{\circ}$, he was put at once into cold water at $60^{\circ}$. The result was, that this almost comatose and dying man shortly became as sensible as any of the bystanders. Now, the effect of such a sudden plunge into cold water must be to drive blood from the surface of the body to the deeper parts, including the brain. Indeed, the treatment is the extreme reverse of that putting of the feet and hands into hot water, which is recommended for cerebral congestion. Yes, doubtless rightly; and if you placed the feet and hands in hot baths, you must tend to draw blood away from a congested brain; and, per contra, by placing not only the feet and hands, but the whole body in very cold water, you must tend to do the opposite of this, and drive blood to the brain. But, in this case, in which every symptom described as cerebral congestion was at its highest degree, and on the point of a fatal issue, the extreme reversal of the treatment for congestion, this driving of blood to the brain wakes up the man in the most magical manner.

Thus the third form of so-called cerebral congestion belies this view of its nature, by passing off under treatment which must add to any congestion really existing in the brain. Let us now look at the last of the forms of so-called cerebral congestion-that noted as delirium. The description is of a patient of advanced age, who, after some hours or even days of undue taciturnity, becomes cheerful, or gay, or hilarious ; he talks loudly and incoherently, but rarely exhibits any violence. He gets out of bed, wanders about his room or ward, opens drawers, puts on his dress, and is bent upon doing something which he cannot explain, or which, if expressed in words, is unnecessary, unaccustomed, and absurd. He is under a delusion of unfixed character, and can usually be directed and controlled without much difficulty. This being the description of the ailment, it may be well to see how far it is a good thing to teach it as a sufficient view that such symptoms are due to congestion of the brain, by giving shortly the history of a case of the kind. Not many months ago, I was asked to see an elderly man whose relatives were said to be quite worn out with taking care of him, on account of his insane conduct. The medical gentleman in charge of his case said that the disorder was congestion of the brain, a bodily disease, and not mental, so that he refused to certify to the patient's insanity. When I saw the man, I found a state of affairs completely justifying this view, according to those who teach that there is a delirious form of congestion of the brain. Mr. P. was fifty-nine years of age. The poor fellow had been knocked down and robbed two years before, and of late he had shown great depression of spirits. Then he grew excited, and talked, and was pleased at the idea of going home. He got out of bed, and wandered about his room, and opened drawers, and dressed himself in the middle of the night, and wanted to go home through the second-floor window or over the balusters of the stairs. But there was no fixed character in his delision; sometimes he was content to stay where he was, under the idea that the Queen had made all the days Sundays now. He was generally submissive, too, and managed without any difficulty, except the fatigue of constantly watching him. He had his wandering 'attacks almost nightly between September and December. This man's disorder corresponded in all points exactly with the so-called delirious form of congestion of the brain; so that the medical practitioner in attendance, trusting his book, was justified in saying that the man had congestion of the brain, and not insanity. But meantime, this madman was endangering his own life very seriously, and causing the gravest overstrain to the members of his family, who were unequal to the care of him. Certificates were immediately signed, and he was put into comparative security, from which the entirely baseless theory of congestion of the brain was keeping him, through the accurate knowledge the doctor had of his teaching. I think one may say, without prejudice, the utterly baseless theory of congestion of the brain. Such excitement does not show congestion of the brain. The best marked cases I have seen of this kind of insane delirium have occurred in heart-disease. Yes; it may be said, exactly the condition to congest the brain. But no one would say that, if he were to bear in mind the kind of hydraulic difficulties that affect the circulation in heart-disease. It is true that, in heart-disease, you have congestions enough; but the congestions occur in the dependent parts of the body, because the inanimate power of gravitation takes hold upon so much of the blood as a weak heart cannot drive, and draws so much blood downwards; but, in doing so, it draws away from the head and other upwardly disposed parts of the body, which are thus kept empty in proportion as the dependent parts are full. And, indeed, in a severe case of delirium, which occurred in a man with cardiac dropsy in Philip Ward of Guy's Hospital, I put the matter to a severe test. The miser. able man, after weeks of breathlessness and sleeplessness, became insane, with the symptoms I have read to you as those of cerebral congestion. I had his pillows taken away, and kept him asleep with chloral for three days. At the end of that time, he was allowed to wake, and he was then quite sane again; and he afterwards recovered so far as to lose his dropsy and leave the hospital. I believe that, if I had kept his head high, he would have died. The symptoms were those of exhaustion in an anæmic brain.

Indeed, the circumstances in which cerebral excitement appear are so far from being of a kind to show an exaggeration of the powers of the circulation, that, from this point of view, we can only wonder how it has come to pass that excitement of mind occurring in the aged, the exhausted, and the dying, is so generally interpreted as signifying an excess of circulation in the brain. The reason, doubtless, is on account of the natural way in which we infer, from the violences set up by a disturbed mind, that a like violence must be at play in the brain, as the organ of the mind. It is so natural to suppose that activity of the eye and tongue and hand must mean activity of the circulation which feeds the nervous centres of the eye and tongue and hand. It is thus that the sight of overactive mental states brings to the medical mind the idea of too great fulness of the blood-vessels, as naturally as the sight of the diurnal course of the sun in the sky brings to the popular mind the idea that the sun goes round the earth. In either case, it is only by further consideration that the fallacy of evidence so apparently obvious is made certain. Reflection will show that the activity of the delurious person may be negative, because it may take its rise through a want of the adjustment of balance of self-restraint, to which a good circulation in a healthy brain gives rise. And the ared and exhausted state of the sufferer points directly to such a negative cause; whilst the treatment also must recognise the negative condition, and do all that can be done to feed the brain and support the system. The decisive style employed by specialists in brain-disease, in their pathological descriptions, has done much to encourage the widely spread delusion as to congestion of the brain. The diseases such as acute mania, for which they seek the anatomical causes, offer a most impressively violent and active behaviour of the brain; and so little appears post mortem, except perhaps a full condition of blood-vessels, that the temptation to dwell upon this state of fulness, and make much of it, has proved irresistible, even when a reasonable doubt of the importance of congestion has forced itself upon their notice. Thus, one of theleast conventional of brainspecialists, Griesinger, after allowing that, in his first edition, the significance of hyperæmia had been greatly exaggerated, goes on, never. theless, with really unabated confidence to assert the usual fallacies. First, he says that, from the changes found after death, increased fulness of the vessels during life is to be assumed. "To this, therefore, a certain connection with the morbid state is to be ascribed"; an obvious fallacy if, by a certain connection, he means a causative connection; and, unless he means a causative connection, his remark has no meaning at all. He then goes on to give the usual account of redness, etc., on the brain, which I have already sufficiently discussed, and ends by saying, "this hyperæmia may easily pass into actual inflammation", a thing which it is utterly impossible that anyone could know. The fact is, that the notion of a flooding of the brain with blood is always ready for use, so that it can be drawn upon by anyone in difficulties for an explanation. And thus, if the alienist needs something to stand for a cause of violent mania, it is congestion of the brain. If the practitioner in charge, when head-disorders do not include madness, wants an explanation of seizures that are the exact reverse of mania, fits in which, instead of violence, you have oppression and stupor, he has it : it is congestion of the brain. If the question is of some "wiry old person"- the expression is not mine-whose circulation is, one would think, too poor to congest anything; if this old person shows negation of brain-activity, still it is congestion of the brain. If a person has drunk enormous quantities of alcohol and is comatose, this, too, is congestion of the brain; and if he has been out in the sun, and has a cerebral seizure, this, too, is congestion of the brain.

I suppose there must be a certain satisfaction in exchanging the 
obscurity that beclouds our ideas of disorder of the nervous system, of whose order we know so little, for a disturbance of the hydraulics of the blood, about which we can know much more; even though the result is only a translation of the terms, in which the symptoms are interpreted to the mind, rather than an explanation of the symptoms. There is, indeed, no true explanation attempted, for no one who asserts that congestion of the brain causes mania, is able to say at all what causes the congestion of the brain.

I have thus far endeavoured to establish these propositions: first, that atmospheric pressure so great as to force blood into the vessels of the brain more thoroughly than could be done by any intrinsic forces of the circulation does not produce cerebral symploms; second, that strangulation and carbonic acid poisoning lend no support to a supposition of independent congestion of the brain; third, that morbid anatomy is unable to show any evidence of general overfilling of the blood-vessels of the brain; fourth, that in the disease described as congestion of the brain, clinical evidence of overfilling of the cerebral blood-vessels is utterly wanting, and that the state of circulation present in these disorders can be shown in typical instances to be the reverse of an overfulness. From all this it follows that there is no pathological justification for our setting up congestion of the brain as a distinct kind of disease, nor for our pretending to explain cerebral symptoms by the assumption of congestion of the brain; for the forcing of blood into the brain whilst the blood is good and properly aërated, and is within its vessels, affects only favourably the functions of the brain as far as our evidence goes. I cannot quite tell where or how the idea arose, but it appears to have been very anciently and naturally supposed that when the senses are lost or submerged, this can only happen through their being drowned in the blood. But the example of sleep is contrary to this rough notion, for Mr. Durham, twenty years ago, showed that the brain is pale in sleep, and other experimenters have since confirmed the observation. We have a daily occurring analogy in every living person which would lead us to expect insensibility to occur with a pale brain, rather than with a brain loaded with blood. Again, fainting teaches us the same lesson : we see in fainting the senses pass into abeyance through bloodlessness of the brain. On the other hand, I do not know of a single proven example in which the senses are lost by pressure of blood within the vessels of the brain. If you object to this, that strangulation gives such an instance, it must be remembered on the contrary that strangulation involves a stoppage of circulation and of all vital changes in the brain; and again, that it must be very thorough to cause unconsciousness. Hence strangulation has no bearing as a fulness of the vessels of the brain; whereas the caissonpressure gives us the most absolute proof that immense pressure of the blood into the cerebral vessels only increases the comfort of the brain, and, in fact, shows that the brain is glad of blood, and the more the better, so long as it is good and does not burst the vessels. I shall now endeavour to point out that bursting of the vessels occurs only in relatively bloodless brains; and so apoplexy has no bearing in favour of congestion of the brain.

The theory of congestion of the brain has been taken from a too simple and superficial notion of the relation of the brain to its bloodsupply. If we take a primitive view of this matter, and suppose the skull to be a closed cavity containing more or less blood, our course of thought would naturally run much in this way. Persons seized with sudden unconsciousness, and dying, are found to have an escape of blood within the brain which compresses it; and other persons attacked in a practically identical way show no such escape of blood. It is, then, in the absence of reasons to the contrary, easy and natural to suppose, as an explanation of all this, that, in the cases where bleeding occurs, the blood before bursting the vessels was pressing very severely inside them, and was distending them and filling the skull overfull, and squeezing the brain very effectually. With such an idea in the mind it is, further, easy to think that, when no bursting of the vessels occurs, this squeezing of the brain by blood in the vessels would cause the same results as the pressure of blood escaping from the vessels when they burst ; and from this point of view it is even possible to regard the bursting of a vessel and the bleeding into the brain as a thing of secondary importance, and to believe that the apoplexy is really set up when a certain engorgement under pressure is reached, which pressure may or may not burst a vessel; but whether it does or no matters little, for the pressure on the brain is the same whether the blood is in or out of the vessels-and, indeed, this opinion has been held and openly stated.

But such a line of reasoning overlooks or underestimates the efficiency of the means whereby the cerebral circulation is relieved, and it overlooks the nature and consequences of arterial tension; and especially it overlooks the fact that apoplexy, when not the result of aneurysms of the cerebral arteries or of cmbolism of those arteries, is nearly always the consequence of the high arterial tension of Bright's disease, and is almost limited to cases of granular kidney and of hypertrophy of the heart. I do not think that the considerations that arise from what we know of sanguineous apoplexy have been reflected upon the naive and simple, but plausible, conception of congestion of the brain, as this conception was formed by our predecessors; for, with what we now know of the circulation, arterial, capillary, and venous, we may prove that the notion of a filling of the vessels of the brain with blood so that they compress the brain is unfounded, so far as sanguineous apoplexy is concerned. The relation of apoplexy to Bright's disease, and through Bright's disease to high arterial tension, throws significant light on the whole of the subject, and proves that the bursting of a small cerebral artery, and the consequent bleeding, does not arise from any general vascular fulness in the cranium, but from tension in the arterial system only, which tension leads to the rupture of the vessel and bleeding. Now, high tension in an artery means resistance to the flow of blood from it into the capillaries, and diminished flow through them into the veins. Hence we see that the high tension in the artery, which eventually bursts it, takes its rise in a cause which prevents supply of blood to the capillary veins, so that the higher tension in the arteries indicates a cause excluding blood from the far more capacious veins; and hence, in proportion as the blood is disposed to burst the artery, in just such proportion is the venous system underfilled. And, in fact, it thus happens that, instead of blood bursting out in apoplexy through a general overfulness of vessels in the skull pressing on the brain, the vascular system as a whole is underfilled.

And this is not a merely theoretical view, although I think the theory itself will stand any examination. But, in fact, before the consideration of the question from a theoretical point of view offered me this explanation of observed facts, I had observed that those brains in which apoplexy occur carry signs of old anæmia, and, indeed, present the appearances characteristic of atrophy from deficient supply. I am $\mathrm{n}$ t here speaking of apoplexy from such accidents as aneurysm or embolism, but of ordinary apoplexy in elderly subjects; and I believe it will be found true that the symptoms which give warning of coming danger--the giddiness and transitory numbness, etc. -are evidences of capillary and venous anæmia of the brain, which is starving under the resistance offered to the entry of a due amount of blood.

Thus, so far from an apoplexy being significant of a general filling to the bursting-point of the intracranial vessels, causing such a fulness under pressure as would compress the brain, it is only in the arteries which are small and not capacious that any excess of blood, or rather excess of tension in the blood, is present; and the idea of a cranium crammed with blood which, if it did not burst the vessels, would equally squeeze consciousness out of the cerebral texture, may be regarded as altogether mythical, and as belonging to bygone and ignorant times.

PAROTID ABSCESS CAUSED BY A FRAGMENT OF HAY. AT the beginning of last October, a healthy young man consulted me about an enlargement of his right parotid gland. The swelling was not very tender, but caused very great discomfort in mastication. I prescribed what I considered suitable remedies, and saw him oc casionally. Weeks passed away, but the swelling and discomfort did not diminish; and about Christmas, finding that he had two decayed and tender molar teeth, I advised him to consult a dentist.

At the end of February my patient came back to me. He had had both his teeth extracted, but his parotid gland was bigger than ever. The tumour had been formerly of the natural colour of the skin, but it was now covered with an inflammatory blush, and seemed to point in one particular spot. A deep puncture was made with a sharp narrow knife, and matter followed the incision. The wound continued to discharge for ten days, and at the end of that time a piece of hay somewhat more than one-third of an inch long escaped, the wound closed, and the swelling subsided. The patient was under treatment altogether just six months.

The explanation of the case now seems simple enough. In the early part of September, he went into the country for his holiday, and he distinctly remembered chewing some hay while he was watching a cow being drenched. A fragment of the hay became lodged between the gum and the cheek, but by working his jaw about, he thought he dislodged and swallowed it. For several days afterwards he had considerable pain in eating, but he attributed this to having sprained the muscles of the jaw in his efforts to dislodge his enemy. A month afterwards, the swelling of the parotid began. There can be no doubt, I think, that the little fragment of hay passed into Stenson's duct when it was thought to have been swallowed, and gradually worked its way up to the salivary gland in front of the ear where it ultimately made its escape. EDward E. MeEres, M.D., Plymouth. 